\title{
A Comparative Study on the Effect of Massularia acuminata Fractions against Bacterial and Fungal Isolates from the Oral Cavity
}

\section{Augustine Olusegun Olusola*, Oluwafemi Shittu Bakare, Oluwafemi Emmanuel Ekun, Adesayo Olufunmi Olusola}

Department of Biochemistry, Faculty of Science, Adekunle Ajasin University, Akungba Akoko, Nigeria

Email: *austinolusola@gmail.com, *augustine.olusola@aaua.edu.ng

How to cite this paper: Olusola, A.O., Bakare, O.S., Ekun, O.E. and Olusola, A.O. (2020) A Comparative Study on the Effect of Massularia acuminata Fractions against Bacterial and Fungal Isolates from the Oral Cavity. Health, 12, 904-913.

https://doi.org/10.4236/health.2020.127067

Received: May 26, 2020

Accepted: July 27, 2020

Published: July 30, 2020

Copyright ( 2020 by author(s) and Scientific Research Publishing Inc. This work is licensed under the Creative Commons Attribution International License (CC BY 4.0).

http://creativecommons.org/licenses/by/4.0/ (c) (i) Open Access

\begin{abstract}
The research aimed at carrying out a comparative study on the effect of Massularia acuminata fractions against bacterial and fungi isolates from the oral cavity. The Antimicrobial assay was carried on the following test organism; Staphylococcus aureus, Klebsiella pnuemoniae, Bacillus subtilis and Escherichia coli and Enterobacter aerogenes. From antifungal result, it was observed that the highest inhibition was observed in the ethanolic extract of Massularia acuminata on Rhyzopus stolonifer, Candida albican and Fusarium solani. The ethanolic and methanolic extracts were also observed from the results to have an intermediate sensitivity to Aspergillus favus, Candida tropicalis, Candida albican, Rhyzopus stolonifer, Fusarium solani, while it was observed to be resistant to Fusarium solani, Rhyzopus stolonifer, Candida tropicalis, Aspergillus favus for all the partitions of the extracts. From the antifungal effect of the $M$. acuminata fractions, it could be inferred that the ethanolic and methanolic extracts were quite effective in the inhibition of the fungi infections and the methanolic extract proved very infective on Candida albican, Rhyzopus stolonifer and Fusarium solani. From the result of the antibacterial effect of $M$. acuminata fractions, the highest inhibition was observed by the methanolic extract of Massularia acuminata against all the Gram negative bacteria and Staphylococcus aureus. Also the aqueous extract also had a less sensitivity against all the test microbes, as all proved resistant. It could be inferred that the fractions of the extracts were unable to inhibit the growth of the strain of gram positive bacteria and probably, the potency could be increased, if the concentrations of the methanolic, ethanolic extracts of Massularia acuminata and the standard drug, tetracycline are increased. Results obtained showed
\end{abstract}


that ethanol was a better and more powerful solvent than water. Whereas, the methanolic extract and the standard drug were quite effective in the inhibition of some gram negative bacteria, Klebsiella pnuemoniae and Enterobacter aerogenes. From the results obtained, it can be deduced that the ethanolic and methanolic extracts of Massularia acuminta possess antifungal and antibacterial potentials, compared with the aqueous extract and its potency as an inhibitor of bacterial and fungi organism found in the oral cavity.

\section{Keywords}

Massularia acuminata, Antimicrobial Assay, Ethanolic and Methanolic Extracts, Tetracycline

\section{Introduction}

Both the upper and lower parts of the human digestive tract harbor a complex ecosystem of bacteria, fungi, protozoa, and viruses, referred to as the microbiome. It begins to form even before birth, in the uterus, developing for another 2 - 3 years after birth to become a stable, fully functioning microbiome, until the physiological changes associated with senescence lead again to substantial shifts in its composition [1] [2] [3]. The lower part of the digestive tract gets "inoculated" every day by about 10 - 11 bacteria from the oral cavity and microbial species detected in the oral and fecal microbiota overlap in about $45 \%$ of tested individuals [4] [5]. Moreover, via the blood stream, these oral bacteria can disseminate all over the body. Fungal microbiota can colonize the gut perorally as well, with some strains detected in the gut likely to be contaminants from the environment or food, rather than commensals [6]. The composition and function of the microbiome change along the digestive tract, from the oral cavity to the rectum. These differences have been previously described in detail [7] and will be briefly outlined below. Collectively, the genes encoded by the microbial genomes outnumber the genes in the human genome about 100 -fold and this variation enables the commensal microbiota to use substrates indigestible by humans [8]. Products of microbial metabolic activity include vitamins, short-chain fatty acids (SCFAs), and other compounds important for host cell metabolism and survival. Moreover, the host's physical interaction with or sensing of the microbial components is important for proper mucosal barrier function and mucosal immune system development and homeostasis. On the other hand, recent studies have shown that some commensal microbes can under certain conditions become pathogenic-so-called pathobionts. Mechanisms include expression of virulence factors, such as adhesion molecules or proteases, or formation of a biofilm, and such activity can lead to disease initiation or progression. One such example is Escherichia coli, a large and diverse group of various bacterial serotypes. E. coli strains differ in their activities and biological roles: some of them are gut commensals and commercially available probiotics, others can be pathogens causing gastroin- 
testinal and urinary infections or pathobionts associated with inflammatory bowel disease (IBD) and colorectal cancer (CRC). The jury is still out on what triggers this transformation of commensals into pathobionts; it might be that a change in the gut microenvironment simply allows the microbes to interact with the host in an aberrant way. The gut microbiota composition and function can be influenced by various factors. For instance, many substances produced by the host and secreted into gut lumen, such as antibacterial peptides, secretory IgA, mucins, cytokines, or neuromediators, can shape the microbial community. Their production depends on host gene polymorphisms and their expression in host cells is often driven by microbial stimulation, creating a positive feedback loop. Mechanisms responsible for cancer development include some of these factors but others are still under scientific investigation. Colorectal cancer is the most prevalent type of cancer in developed and developing countries [9]. Only a small percentage of colorectal cancers is hereditary or associated with certain predisposing conditions, such as chronic intestinal inflammation in IBD patients. The majority of cases thus represents sporadic cancers (85\% - 95\%) and can be, to some extent, influenced by environmental factors. The composition and metabolic activity of gut microbiota may be therefore a crucial component of CRC pathogenesis [10]. Findings of significant colonization of cancer tissue by microbes usually found in the oral cavity have sparked a debate about a possible involvement of oral microbiota in CRC development process. Many experimental studies have provided evidence for a significant role of microbiota in carcinogenesis. However, due to the complexity of microbial cooperation and interaction with the host, all the underlying mechanisms are yet to be elucidated. Here, we evaluated the antifungal and antibacterial potentials of ethanolic and methanolic extracts of Massularia acuminata in comparison with the aqueous extract and its potency as an inhibitor of bacteria and fungi found in the oral cavity.

\section{Materials and Methods}

\subsection{Collection and Extraction of Plant Materials}

The plant parts: stem barks of Massularia acuminata were collected in Ute, in Owo Local Government area of Ondo State. The authentication were done in Department of Biotechnology, Adekunle Ajasin University, Akungba Akoko and re-authenticated at IFE Herbarium at Obafemi Awolowo University, Ile Ife. The stem bark were air-dried and crushed into fine powder. The powdered samples were extracted separately with methanol, ethanol and butanol with continuous agitation; the extracts were concentrated at $35^{\circ} \mathrm{C}$ with rotary evaporator and water bath to dryness. A known amount of pulverized sample of Massularia acuminata $(500 \mathrm{~g})$ were percolated in 1 litre of distilled water and respective alcoholic solvents; with ratio of powder and solvent of 1:2, and with intermittent shaking and kept in the refrigerator for 48 hours. The solution were thereafter filtered using Whatman No. 1 filter paper and the filterate concentrated on a water bath to give a yield of $25.50 \mathrm{~g}$ representing a percentage yield of $5.10 \%$. 


\subsection{Equipment and Apparatus}

Bench top centrifuge machine, refrigerator, micropipette, spectrophotometer, standard mouse cage, water Bath and sensitive weighing balance. Beaker $(50 \mathrm{ml}$ and $1000 \mathrm{ml}$ ), needle and syringe, measuring cylinder, spatula, dissecting kit, intubator, test tubes, test tube rack and reagent bottle.

\subsection{Materials}

Permanent marker, masking tape, disposable hand gloves, cotton wool, foil paper, tissue paper, EDTA bottles, plain bottles and lithium heparin bottle.

\subsection{Chemicals and Reagents}

Distilled water, hand sanitizer, antiseptic, normal saline and aluminium chloride.

\subsection{Methods}

\subsubsection{Microorganisms and Culture Conditions}

The bacterial strains used are Grams positive (Staphylococcus aureus, and Bacillus substilis) and Gram negative (Escherichia coli, Klebsiella pnuemoniae, Enterobacter aerogenes), was obtained from microbiology department, Adekunle Ajasin University, Akunga Akoko, Ondo state and was cultured aerobically at $37^{\circ} \mathrm{C}$ in nutrient agar medium. Before experimental use, cultures from solid medium will be sub-cultivated in liquid media, incubated for 24 hours and was used as source of inoculums for each experiment. Antimicrobial activity was measured using agar-well diffusion method.

\subsubsection{Antimicrobial Screening of Massularia acuminata Crude Extracts on Selected Pathogens}

Antibacterial activities of the plant extracts of Massularia acuminata and fractionated constituents were tested by disc-diffusion method. The minimal inhibition concentration (MIC) of the extracts was determined. Five bacterial strains, Staphylococcus aureus, Bacillus substilis, Escherichia coli,Klebsiella pnuemoniae, and Enterobacter aerogenes were used in this study. The organisms were obtained from microbiology Department, Adekunle Ajasin University, Akungba Akoko, Ondo state and were cultured aerobically at $37^{\circ} \mathrm{C}$ in nutrient agar medium. The bacteria were tested for purity by culturing on nutrient agar and maintained on nutrient agar slants.

\subsubsection{Antibacterial Activity of the Extracts}

Susceptibility of bacteria isolate to plant extract were determined following the BSAC Diffusion Method for Antimicrobial Susceptibility Testing Version 9.1 [11]. This test was carried out to determine the antimicrobial ability of the plant extract to inhibit the growth of the bacteria isolate that was collected from Microbiology Department FUTA. The plate diffusion technique of [12] was used for the antibiotic sensitivity test. Overnight cultures of the organisms were swabbed 
on sterile Muller Hilton solidified Agar plates using sterile swab sticks. $8 \mathrm{~mm}$ sized corn borer was used to bore hole on the agar surface at equidistance the well was filled with the diluted plant extract, a known antibiotic was used as positive control while distilled water was used as negative control. All the plates were incubated at $37^{\circ} \mathrm{C}$ to 24 hours. The zones of inhibition generated by the antibiotics were measured to the nearest millimeters $(\mathrm{mm})$ and interpreted as sensitive (S), Intermediate (I) and resistant (R). The zones of inhibition were measured and interpreted according to [13]. The zones of inhibition were compared with that of Nystatin and tetracycline.

\subsubsection{Determination of the Minimum Inhibitory Concentration (MIC)}

Five concentrations of each extract $(40,60,80$ and $100 \mathrm{mg} / \mathrm{ml})$ was prepared. The antimicrobial effect of each concentration was measured. The various concentrations was loaded onto $6 \mathrm{~mm}$ disks which was then pressed onto already prepared Mueller-Hinton agar plates and SDA plates. The inoculated plates were incubated at $35^{\circ} \mathrm{C}$ for $48 \mathrm{~h}$. MICs were determined after 24 hours for the bacteria and after 48 hours for Massularia acuminata and its fractions extract zones of inhibition were measured at the end of the incubation period. The MICs was determined as the lowest concentrations of extracts inhibiting the visible growth of each organism on the agar plate.

\subsubsection{Disc Diffusion Assay}

The strains of microorganism obtained were inoculated in conical flask containing $100 \mathrm{ml}$ of nutrient broth. These conical flasks were incubated at $37^{\circ} \mathrm{C}$ or $24 \mathrm{~h}$ and were referred to as seeded broth. Media were prepared using Muller Hinton Agar (Himedia), poured on Petri dishes and inoculated with the test organisms from the seeded broth using cotton swabs. Sterile discs of six millimeter with had been impregnated with $30 \mu \mathrm{l}$ of test extract and introduced onto the upper layer of the seeded agar plate. The plates were incubated overnight at $37^{\circ} \mathrm{C}$. The antimicrobial activity was recorded by measuring the width of the clear inhibition zone around the discs using zone reader $(\mathrm{mm})$. Tetracycline $(100 \mu \mathrm{g} / \mathrm{disc})$ were used as standards.

\subsubsection{Statistical Analysis}

Experimental results were expressed as mean \pm standard error of mean (SEM). All measurements were replicated three times. The results were analyzed using one way analysis of variance (Anova). The level of significance used was $\mathrm{P}<$ 0.05 .

\section{Results}

\subsection{Anti-Fungal Activity of M. acuminata Fractions}

Table 1 shows the antifungal effects of different solvent extracts of $M$. acuminate plant. All values are expressed as mean inhibition zones $(\mathrm{mm}) \pm$ SEM of three replicates $(\mathrm{mm})$. 


\subsection{Anti-Bacterial Activity of M. acuminata Fractions}

Table 2 shows the antibacterial effects of different solvent extracts of $M$. acuminate plant. All values are expressed as mean inhibition zones $(\mathrm{mm}) \pm \mathrm{SEM}$ of three replicates $(\mathrm{mm})$.

\subsection{Minimum Inhibitory Concentration (MIC) of M. acuminata Fractions}

Table 3 shows the minimum inhibitory concentration (MIC) of methanolic extract, ethanolic extract and aqueous extract of Massularia acuminata against test microbes $(\mathrm{mm})$.

Figure 1 shows petri dishes indicating zones of inhibition by various alcoholic and aqueous extracts of Massularia acuminate; a clear zones between the organism and the holes filled with bacterial strains; the bacterial or fungal strain is susceptible to the antimicrobial agent, with an appearance of a clear zone of inhibition on the agar plates.

Table 1. Anti-fungal activity of $M$. acuminata fractions.

\begin{tabular}{ccccc}
\hline Fungi organism & $\begin{array}{c}\text { Methanolic } \\
\text { M. acuminata } \\
\text { extract }\end{array}$ & $\begin{array}{c}\text { Aqueous } \\
\text { acuminata } \\
\text { extract }\end{array}$ & $\begin{array}{c}\text { M. acuminata } \\
\text { extract }\end{array}$ & $\begin{array}{c}\text { Nystatin } \\
(\mathrm{mm})\end{array}$ \\
\hline Aspergillus favus & $17.66 \pm 0.19$ & $10.16 \pm 0.09$ & $14.33 \pm 0.19$ & $21.2 \pm 0.11$ \\
Candida tropicalis & $16.55 \pm 0.17$ & $12.2 \pm 0.11$ & $14.5 \pm 0.23$ & $23.23 \pm 0.13$ \\
Candida albican & $16.26 \pm 0.15$ & $11.33 \pm 0.19$ & $22.1 \pm 0.05$ & $22.1 \pm 0.05$ \\
Rhyzopus stolonifer & $16.4 \pm 0.15$ & $12.43 \pm 0.17$ & $25.2 \pm 0.24$ & $26.73 \pm 0.21$ \\
Fusarium solani & $14.46 \pm 0.16$ & $12.5 \pm 0.16$ & $23.46 \pm 0.26$ & $23.33 \pm 0.19$ \\
\hline
\end{tabular}

Sensitive $(S) \geq 21$, Intermediate $(\mathrm{I}) \leq 20$ and resistant $(\mathrm{R}) \leq 14$.

Table 2. Anti-bacterial activity of $M$. acuminata fractions.

\begin{tabular}{ccccc}
\hline Bacterial Strain & $\begin{array}{c}\text { Methanolic } \\
\text { M. acuminata } \\
\text { extract }\end{array}$ & $\begin{array}{c}\text { Aqueous } \\
\text { acuminata } \\
\text { extract }\end{array}$ & $\begin{array}{c}\text { M. acuminata } \\
\text { extract }\end{array}$ & $\begin{array}{c}\text { Tetracycline } \\
30 \mu \mathrm{g}\end{array}$ \\
\hline Gram positive & $10.63 \pm 0.18$ & $0.00 \pm 0.00$ & $11.33 \pm 0.50$ & $12.66 \pm 0.19$ \\
Bacillus subtilis & $13.16 \pm 0.25$ & $0.83 \pm 0.03$ & $12.16 \pm 0.34$ & $13.33 \pm 0.19$ \\
Staphylococcus aureus & & & & \\
Gram negative & $13.33 \pm 0.50$ & $0.86 \pm 0.01$ & $11.43 \pm 0.25$ & $13.66 \pm 0.38$ \\
Escherichia coil & $14.66 \pm 0.38$ & $11.66 \pm 0.50$ & $12.23 \pm 0.13$ & $14.26 \pm 0.21$ \\
Klebsiella pnuemoniae & & & $12.33 \pm 0.19$ & $14.33 \pm 0.19$ \\
Enterobacter aerogenes & $14.33 \pm 0.50$ & $0.86 \pm 0.01$ & & \\
\hline
\end{tabular}

Sensitive $(S) \geq 21$, intermediate $(\mathrm{I}) \leq 20$ and resistant $(\mathrm{R}) \leq 14$. 
Table 3. Minimum inhibitory concentration (MIC) of M. acuminata fractions.

\begin{tabular}{ccccccc}
\hline $\begin{array}{c}\text { Plant } \\
\text { Extract }\end{array}$ & $\begin{array}{c}\text { Conc } \\
\mathrm{mg} / \mathrm{ml}\end{array}$ & B. subtilis & S. aureus & E. coli & K. pnuemoniae & E. aerogenes \\
\hline $\begin{array}{c}\text { Methanolic } \\
\text { Extract }\end{array}$ & 100 & $10.53 \pm 0.16$ & $13.83 \pm 0.25$ & $13.63 \pm 0.18$ & $14.53 \pm 0.16$ & $14.76 \pm 0.35$ \\
& 80 & $10.35 \pm 0.17$ & $0.86 \pm 0.01$ & $0.86 \pm 0.01$ & $0.76 \pm 0.05$ & $0.83 \pm 0.03$ \\
& 60 & $0.00 \pm 0.00$ & $10.55 \pm 0.16$ & $0.86 \pm 0.01$ & $11.16 \pm 0.09$ & $10.23 \pm 0.13$ \\
& 40 & $0.00 \pm 0.00$ & $0.00 \pm 0.00$ & $0.00 \pm 0.00$ & $0.00 \pm 0.00$ & $0.00 \pm 0.00$ \\
Ethanolic & 100 & $10.28 \pm 0.13$ & $11.7 \pm 0.20$ & $11.8 \pm 0.35$ & $11.23 \pm 0.0 .13$ & $12.4 \pm 0.17$ \\
Extract & 80 & $0.83 \pm 0.03$ & $10.66 \pm 0.19$ & $0.86 \pm 0.01$ & $0.80 \pm 0.03$ & $11.16 \pm 0.41$ \\
& 60 & $0.00 \pm 0.00$ & $0.00 \pm 0.00$ & $0.00 \pm 0.00$ & $0.00 \pm 0.00$ & $0.00 \pm 0.00$ \\
& 40 & $0.00 \pm 0.00$ & $0.00 \pm 0.00$ & $0.00 \pm 0.00$ & $0.00 \pm 0.00$ & $0.00 \pm 0.00$ \\
Aqueous & 100 & $0.00 \pm 0.00$ & $0.86 \pm 0.01$ & $0.83 \pm 0.03$ & $0.83 \pm 0.03$ & $0.86 \pm 0.01$ \\
Extract & 80 & $0.83 \pm 0.03$ & $0.86 \pm 0.01$ & $0.00 \pm 0.00$ & $0.00 \pm 0.00$ & $0.00 \pm 0.00$ \\
& 60 & $0.00 \pm 0.00$ & $0.00 \pm 0.00$ & $0.00 \pm 0.00$ & $0.00 \pm 0.00$ & $0.00 \pm 0.00$ \\
& 40 & $0.00 \pm 0.00$ & $0.00 \pm 0.00$ & $0.00 \pm 0.00$ & $0.00 \pm 0.00$ & $0.00 \pm 0.00$ \\
\hline
\end{tabular}

Sensitive $(\mathrm{S}) \geq 21$, Intermediate $(\mathrm{I}) \leq 20$ and resistant $(\mathrm{R}) \leq 14$ Intermediate $(\mathrm{I}) \leq 20$.

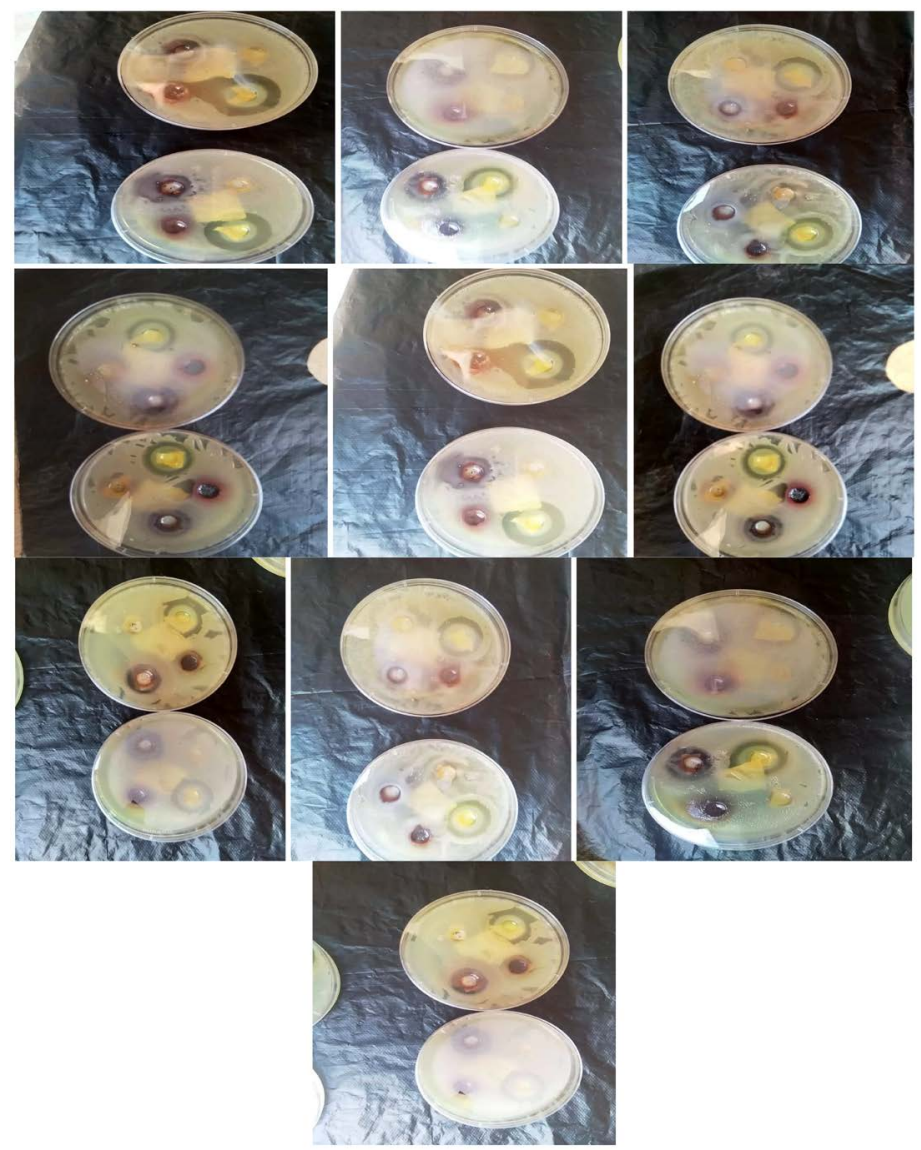

Figure 1. Petri dishes showing zones of inhibition by various alcoholic and aqueous extracts of Massularia acuminate. 


\section{Discussion}

People are aware that good oral health is very important. Consequently, daily brushing and flossing are taught to us from a very early age to keep our teeth as clean as possible. However, not everyone is aware that good oral health is more than pretty white of teeth. Both the upper and the lower parts of the human digestive tract harbor a complex ecosystem of bacteria, fungi, protozoa and viruses, referred to as the microbiome [3]. It begins to form even before birth, in the uterus, developing for another 2 - 3 years after birth to become a stable, fully functioning microbiome, until the physiological changes associated with senescence lead again to substantial shifts in its composition [1] [2].

The results illustrated the inhibitory effect of the various fractions of Massularia acuminata extracts on the respective opportunistic infection found in the oral cavity as seen in the results. Opportunistic infections are infections that occur more often or are more severe in people with weakened immune systems than in people with healthy immune systems.

From the antifungal effect of the M. acuminata fractions, it could be inferred that the ethanolic and methanolic extracts were quite effective in the inhibition of the fungi infections and the methanolic extract proved very infective on Candida albican, Rhyzopus stolonifer and Fusarium solani. The degree of inhibition can be increased if, the concentrations of both extracts are increased. The aqueous extract was unable to inhibit the infections and probably, it effectiveness could be improved if its concentrations is increased. The standard drug, Nystatin, which has a broad spectrum in the inhibition of several opportunistic infections proved very much infective in the inhibition of the fungi infections.

From the result of the antibacterial effect of $M$. acuminata fractions, it could be inferred that the fractions of the extracts were unable to inhibit the growth of the strain of gram positive bacteria and probably, the potency could be increased, if the concentrations of the methanolic, ethanolic extracts and the standard drug, tetracycline are increased. Whereas, the methanolic extract and the standard drug were quite effective in the inhibition of some gram negative bacteria, Klebsiella pnuemoniae and Enterobacter aerogenes, and its effectiveness could be increased, if the concentrations are increased. The ethanolic and aqueous extracts were unable to inhibit the growth of the bacteria.

Massularia accuminata showed a high inhibitory activity against the bacteria species tested, since it exhibited the highest antibacterial inhibition. This was in conformity with the work by [14]. Results obtained showed that ethanol was a better and more powerful solvent than water. This is in agreement with the report of [15], which stated that active components of plants are more soluble in organic solvent. The high potency of the ethanol extract may be attributed to the dissolving power of alcohol over water [16]. The antifungal and antibacterial inhibitory effects showed by the chewing stick extracts can be attributed to the presence of phytochemical compounds (glycosides, alkaloids, saponins, tannins, flavonoids, anthocyanins, anthraquinones and phlobatannins) present in them. 
This is also in agreement with the work reported by [17] [18].

From the result, illustrating Minimum Inhibitory concentration (MIC) of methanolic extract, ethanolic extract and aqueous extract of Massularia acuminata against test microbes, it could be inferred that at high concentrations the inhibitory effects of various fractions of the extracts increased when compared with lower concentrations of the extracts. Probably, the effectiveness of the extracts could be improved, if the concentrations of the extracts are increased.

\section{Conclusion}

In conclusion, from the results obtained, it can be deduced that the ethanolic and methanolic extracts of Massularia acuminta possess antifungal and antibacterial potentials, when compared with the aqueous extract and its potency as an inhibitor of bacteria and fungi found in the oral cavity can be increased, if their respective concentrations are increased, provided the extracts have not gotten to their lethal doses, where they could prove fatal.

\section{Acknowledgements}

This research was funded by Tertiary Education Trust Fund (TET Fund) through its Institutional Based Research Grant program. We are also grateful to Adekunle Ajasin University Akungba Akoko, Ondo State, Nigeria, for providing the enabling environment for the sponsorship and success of the work.

\section{Conflicts of Interest}

The authors declare no conflicts of interest regarding the publication of this paper.

\section{References}

[1] Alderberth, I. and Wold, A.E. (2009) Establishment of the Gut Microbiota in Western Infants. Acta Paediatrica, 98, 229-238. https://doi.org/10.1111/j.1651-2227.2008.01060.x

[2] Aagaard, K., Ma, J., Anthony, K.M., Ganu, R. and Petrosino, J. (2014) The Placenta Harbors a Unique Microbiome. Science Translational Medicine, 6, $273 \mathrm{ra} 65$. https://doi.org/10.1126/scitranslmed.3008599

[3] Maffei, V.J., Kim, S., Blanchard, E., Luo, M., Jazwinski, S.M. and Taylor, C.M. (2017) Biological Aging and the Human Gut Microbiota. The Journals of Gerontology Series A Biological Sciences and Medical Sciences, 2, 147-1482. https://doi.org/10.1093/gerona/glx042

[4] Socransky, S.S. and Haffajee, A.D. (2005) Periodontal Microbial Ecology. Periodontology, 38, 135-187. https://doi.org/10.1111/j.1600-0757.2005.00107.x

[5] Segata, N., Waldron, L., Ballarini, A., Narasimhan, V., Jousson, O. and Huttenhower, C. (2012) Metagenomic Microbial Community Profiling Using Unique Clade-Specific Marker Genes. Nature Methods, 9, 811-814. https://doi.org/10.1038/nmeth.2066

[6] Trojanowska, D., Zwolinska-Wcislo, M., Tokarczyk, M., Kosowski, K., Mach, T. and Budak, A. (2010) The Role of Candida in Inflammatory Bowel Disease. Estima- 
tion of Transmission of $C$. albicans Fungi in Gastrointestinal Tract Based on Genetic Affinity between Strains. Medical Science Monitor, 16, 451-457.

[7] Arumugam, M., Raes, J., Pelletier, E., Pastier, D.L., et al. (2011) Enterotypes of the Human Gut Microbiome. Nature, 473, 174-180.

https://doi.org/10.1038/nature09944

[8] Qin, J., Li, Y., Cai, Z., Li, S., Zhu, J., Zhang, W., Guan, Y. and Shen, D. (2012) A Metagenome-Wide Association Study of Gut Microbiota in Type 2 Diabetes. Nature, 490, 55-60. https://doi.org/10.1038/nature11450

[9] Ferlay, J., et al. (2015) Cancer Incidence and Mortality Worldwide: Sources, Methods and Major Patterns in GLOBOCAN 2012. International Journal of Cancer, 136, E359-E386. https://doi.org/10.1002/ijc.29210

[10] Arthur, J.C. and Jobin, C. (2011) The Struggle within: Microbial Influences on Colorectal Cancer. Inflammatory Bowel Diseases, 17, 396-409. https://doi.org/10.1002/ibd.21354

[11] Andrews, J.M. (2009) BSAC Standardized Disc Susceptibility Testing Method (Version 8). Journal of Antimicrobial Chemotherapy, 64, 454-489. https://doi.org/10.1093/jac/dkp244

[12] Willey, J.M., Sherwood, L.M. and Woolverton, C.J. (2008) Prescott, Harley and Klein's Microbiology. 7th Edition, McGraw Hill, New York.

[13] NCCLS (2000) Performance Standards for Antimicrobial Disk Susceptibility Tests. Approved Standards 7th Edition, NCCLS Document M2-A7. NCCLS, Wayne.

[14] Barnabas, C.G. and Nagarajan, S. (1998) Antimicrobial Activity of Flavonoids of Some Medicinal Plants. Fitoterapia, 3, 508-510.

[15] Kareem, S.O., Akpan, I. and Ojo, O.P. (2008) Antimicrobial Activities of Calotropisprocera on Selected Pathogenic Microorganisms. African Journal of Biomedical Research, 11, 105-110. https://doi.org/10.4314/ajbr.v11i1.50674

[16] Majorie, M.C. (1999) Plant Products as Antimicrobial Agents. Clinical Microbiological Review, 12, 564-582. https://doi.org/10.1128/CMR.12.4.564

[17] Cowan, M.M. (1999) Plant Products as Antimicrobial Agents. Clinical Microbiological Review, 12, 564-582. https://doi.org/10.1128/CMR.12.4.564

[18] Draughon, F.A. (2004) Use of Botanicals as Bio-Preservatives in Foods. Food Technology, 58, 20-28. 